\title{
Localization of electrocutaneous stimuli on the fingers and forearm: Effects of electrode configuration and body axis
}

\author{
ATSUKI HIGASHIYAMA and MAMORU HAYASHI \\ University of Osaka Prefecture, Sakai, Japan
}

\begin{abstract}
Four experiments were done on the effects of electrode configuration (concentric vs. unifocal) and body axis (longitudinal vs. transverse) on localization of electrocutaneous pulse stimuli at the fingers and forearm. Subjects pointed to the apparent location of current pulses. For the transverse placement of electrodes, pulses were localized correctly, whatever the configuration and the body site might be. In addition, intrasubject variability at the forearm was smaller for the transverse axis than for the longitudinal axis. For the longitudinal placement of electrodes, pulses were localized as a function of configuration and body site. At the fingers, concentric electrodes provided precise localization but unifocal electrodes provided a great mislocalization; and intrasubject variability of localization was larger for the unifocal electrodes than for the concentric electrodes. At the forearm, whatever the configuration might be, the pulses were localized more proximally than the stimulus site; and intrasubject variability of localization did not differ between the configurations. These results are related to Boring's anchor theory, apparent distance between two points, and the localization of other somatosensory stimuli.
\end{abstract}

In this paper, we will be concerned with the localization of electrocutaneous stimuli that are presented in either of two typical electrode configurations. One configuration involves concentric electrodes as used by Békésy (1959a, 1959b), Marks, Girvin, O'Keefe, et al. (1982), Marks, Girvin, Quest, et al. (1982), Saunders (1973), Solomonow, Lyman, and Freedy (1977), and Tursky and colleagues (Tursky, 1973; Tursky \& O'Connell, 1972; Tursky, Watson, \& O'Connell, 1965). In this configuration, the center electrode is separated from the surrounding one by a distance of several millimeters or less. In much of his work, Gibson (1968) placed a large anodal electrode under the sole of the foot and placed the small cathodal electrode(s) at the forearm where the stimuli could be localized. In this configuration, therefore, the distance between the anode and the cathode can be $1 \mathrm{~m}$ or more. The electrodes in this configuration have been called monopolar (Gibson, 1968) or unifocal (Girvin et al., 1982). There are configurations intermediate between the two. Jones (1956) and Wieland (1960) placed a large anode on the ventral surface of the forearm and a small cathode(s) on its dorsal surface. Anderson and Munson (1951), Higashiyama and Rollman (1991), and Higashiyama and Tashiro (1983) placed two same-sized electrodes side by side on the forearm. In these configurations, the distance between the electrodes usually ranges from 2 to $20 \mathrm{~cm}$.

We thank two anonymous reviewers who improved the English of an earlier draft. M. Hayashi is now at Digital Equipment Corporation Japan. Correspondence should be addressed to A. Higashiyama, Psychology Laboratory, College of Integrated Arts and Sciences, University of Osaka Prefecture, Sakai 593, Japan.
Although the effects of electrode configuration on localization have not been systematically studied, it is possible to compare the electrode configurations in terms of other aspects of spatial sensitivity to current. Concentric electrodes may yield smaller two-point thresholds (i.e., a distance between two stimulus points that are barely perceived as being separate) than do unifocal electrodes. Solomonow and colleagues (Solomonow \& Lyman, 1980; Solomonow et al., 1977) reported a threshold of about $9 \mathrm{~mm}$ with concentric electrodes on the forearm, whereas the results of Jones (1956) and Wieland (1960) suggested that a threshold of $10 \mathrm{~cm}$ or more would be obtained with electrodes separated by several centimeters. To produce phantom sensation and apparent movement, Békésy (1959a, 1959b, 1967, p. 123) recommended concentric electrodes rather than unifocal electrodes, because concentric electrodes make it possible to reduce stray currents and to define the locus of the stimulus. Subsequent studies have indeed demonstrated apparent movement with concentric electrodes at the thigh (Sherrick \& Rogers, 1966) or with a similar means at the fingers (Kirman, $1974,1975)$. Nevertheless, Gibson (1968) succeeded in observing these phenomena with unifocal electrodes at the forearm.

In the present study, we examined localization with current at the fingers and the forearm. Although this has not been studied extensively, the findings obtained with tactile stimulation can provide a prediction regarding electrocutaneous localization. Weber (1834/1978) and Weinstein (1968) demonstrated that the body site affects spatial sensitivity (the two-point threshold and the error of localization) for tactile stimulation, and that the error of localization (i.e., the difference between the stimulus site and the 
pointed site) is smaller on the fingers than it is on the forearm. This finding can be explained in terms of receptor density: the greater the density of receptors, the smaller the error of localization (Békésy, 1967, p. 58). If the same line of reasoning holds for electrocutaneous stimulation, the error of localization should be smaller on the fingers than on the forearm, since the fingers have a much greater density of innervation than does the forearm. The same prediction can be obtained from Vierordt's law, which states that the two-point threshold for tactile stimulation is lower at mobile body sites than it is at less mobile sites (Boring, 1942, p. 478). Since there is a high correlation ( $r=.92$ ) between the two-point threshold and the error of localization (Cholewiak \& Collins, 1991; Sherrick \& Cholewiak, 1986), the error of localization should be smaller at more mobile sites.

The localization of current may also be affected by the body axis. There are two fundamental body axes-the longitudinal and transverse axes. The longitudinal axis passes through the body parallel to the limbs; the transverse axis passes through the body, intersecting the limbs at right angles. Since, with tactile stimulation, the body axis affects localization, the two-point threshold (Boring, 1930; Weber, 1834/1978; Weinstein, 1968), and the perceived distance between two stimuli (Green, 1982), it can be supposed that there are several anisotropic properties of current stimulation as well. According to a short report by Gilmer (1961), the two-point threshold for current on the forearm was on the order of millimeters for the transverse direction and inches for the longitudinal direction. Marks, Girvin, Quest, et al. (1982) found that the distance between two electrical stimuli on the forearm is perceived as larger in the transverse direction than in the longitudinal direction. However, this anisotropy was limited to distances of $20 \mathrm{~mm}$ or more and was not found for the abdomen. Sparks (1979) suggested that the ability to identify the direction of electrocutaneous stimulation along linear multistimulator arrays does not differ between the longitudinal and transverse axes of the abdomen. However, we have not found much literature that is directly related to the effects of the body axis on the localization of electrical stimuli.

Our experiments were conducted on the volar surface of the index, middle, and ring fingers (Experiments 1A-C) and on the volar surface of the forearm (Experiment 2). In all the experiments, the subjects pointed to the apparent location of the pulses that were delivered to electrodes. In Experiment 1A, three concentric or unifocal electrodes were placed in a row on the middle finger (longitudinal condition) or on the volar tips of the index, middle, and ring fingers (transverse condition). In Experiment 1B, which was an extention of Experiment 1A, three concentric or unifocal electrodes were placed in a row on each volar surface of the index, middle, and ring fingers. In Experiment 1C, we examined whether apparent location would be affected by the subjects' viewing the electrode positions. In Experiment 2, seven concentric or unifocal electrodes were placed longitudinally or transversely on the forearm.

\section{EXPERIMENT 1A}

\section{Method}

Subjects. Twenty-nine undergraduates (17 males and 12 females) volunteered as subjects. No special qualifying criteria were imposed.

Apparatus. A constant-current stimulator (Nihonkoden SEN-7103 and SS-102J) was used to provide electrical monophasic positive pulses to the subject's skin through paired silver electrodes. A system comprising an Apple II microcomputer and a Sanwa time regulator determined the time schedule of warning buzzer, foreperiod, and intertrial interval. A trial sequence was started with a warning buzzer, followed by single or multiple pulses to the electrodes after a foreperiod of $2.16,3.47$, or $4.81 \mathrm{sec}$. The foreperiod was randomized for each trial. The intertrial interval was approximately $10 \mathrm{sec}$. The current delivered by this apparatus was limited to $10 \mathrm{~mA}$, to protect the subjects from accidents. Further details of the apparatus are provided elsewhere (Higashiyama \& Tashiro, 1988,1990 ).

Electrode placement. Two types of electrode configurations were examined. The first one was unifocal; an anodal electrode $30 \mathrm{~mm}$ in diameter $\left(706 \mathrm{~mm}^{2}\right)$ was placed on the instep of the right foot, and six cathodal electrodes, each of which was $7 \mathrm{~mm}$ in diameter $\left(38 \mathrm{~mm}^{2}\right)$, were placed on the right fingers and wrist, as shown in Figure 1. The experimenter activated one cathodal electrode at a time with a switch that controlled the stimulator and the electrodes.

The second type of electrode pair resembled the concentric configuration used by Solomonow and Lyman (1980). The cathode was an inner disk $3.3 \mathrm{~mm}$ in diameter $\left(8.5 \mathrm{~mm}^{2}\right)$ and the anode was an outer ring $6.6 \mathrm{~mm}$ in diameter outside and $5.2 \mathrm{~mm}$ in diameter inside $\left(13.0 \mathrm{~mm}^{2}\right)$. The disk was thus separated from the ring by about $1 \mathrm{~mm} .{ }^{1}$ A pair of concentric electrodes was placed at each of the six sites shown in Figure 1. For both configurations, the wrist electrode(s) was (were) used to measure the threshold, and the finger electrodes were used for electrocutaneous localization.

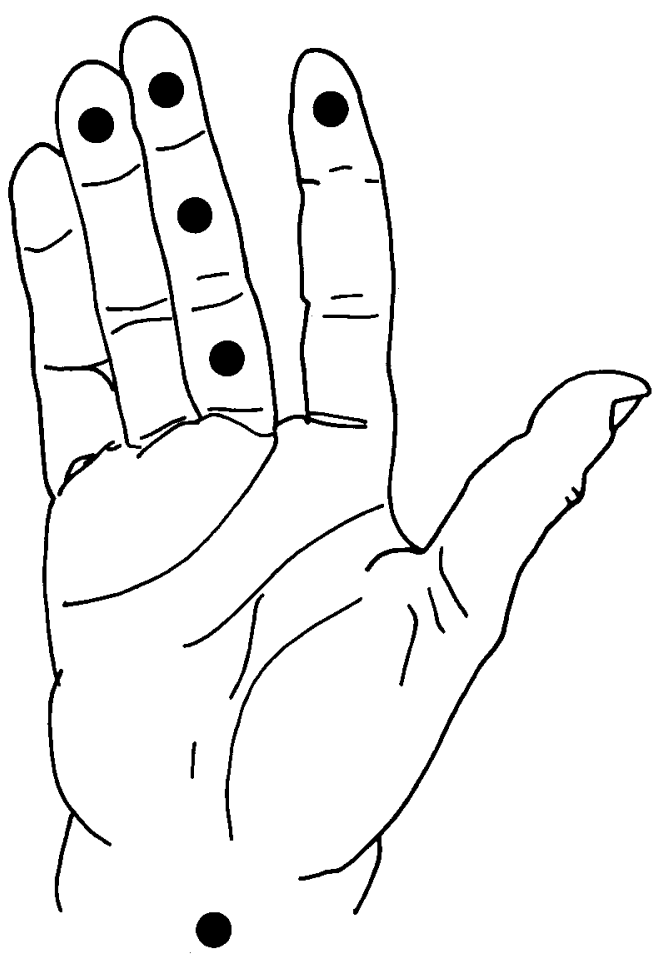

Figure 1. Six electrodes placed on the fingers and wrist. 
Procedure. Thirteen of the subjects received only the unifocal electrodes, and the remaining 16 subjects received only the concentric electrodes. Each subject was seated with the right hand resting on the table, and the hand was washed with an alcohol solution. For the unifocal electrodes, each electrode was filled with keratin electrode paste and was attached by a piece of semitransparent surgical tape. For the concentric electrodes, the keratin paste was not used, because the separation between two electrodes was so narrow that the paste patches overflowing from the electrodes could come into contact with each other and cause a local short circuit (Higashiyama \& Tashiro, 1983). Instead of keratin paste, the skin surface was rubbed with Nihonkoden SkinPure gel, which consists of sea sand and wetting agent, to reduce the skin impedance. The electrodes were then attached with tape directly to the skin.

An absolute threshold for 2-msec single pulses was determined for the wrist electrode with a staircase procedure. Each trial sequence was started with a warning buzzer. After a foreperiod of $2.16,3.47$, or $4.81 \mathrm{sec}$, a single pulse was delivered to the electrodes. The subject was asked to judge whether current was present on the wrist. When the current was detected, it was decreased by a step (about $.08 \mathrm{~mA}$ ); when it was not, it was increased by a step. Each subject made more than 32 "yes" /"no" judgments after several preliminary trials. A current pulse was presented on every trial; there were no blank trials. The mean threshold for the concentric electrodes $(M=5.96 \mathrm{~mA}, S D=1.41 \mathrm{~mA})$ was significantly higher than that for the unifocal electrodes $(M=1.48 \mathrm{~mA}, S D=$ $.52 \mathrm{~mA})[t(27)=13.4, p<.001$; see the Appendix $] .^{2}$

After determining the threshold for the wrist, the experimenter delivered the wrist threshold current to each finger electrode. The wrist threshold current was used first to guard the subject from the shock that could be caused by high current. The sensation threshold for current depends on the subject, and since the dynamic range of current is narrow, the same current pulses can produce touch for some subjects and pain for other subjects. The wrist threshold current was not usually perceived for any finger, because of the higher finger threshold. The experimenter then increased the finger current gradually and determined a current that was easy to perceive but was neither unpleasant nor painful. In addition, the instructions emphasized that the subjective intensity of the pulses should be the same among the finger electrodes. The subject directed the experimenter to increase and/or decrease the current and to report when the pulses were perceived to be equal to each other. Through this successive comparison, the current for the localization task was determined for each finger electrode.

In the localization task, the subject was asked to point to the apparent location of each pulse. To facilitate the subject's responses, a list of body sites was presented. It included (1) the tip of the middle finger, (2) the center of the middle finger, (3) the base of the middle finger, (4) the tip of the index finger, (5) the tip of the ring finger, (6) the wrist, and (7) the instep of the foot. When a pulse was perceived at a site other than these, the subject was asked to point to its place with the index finger of the left hand. In pointing to this apparent location, the subject was prevented from touching the place where he/she perceived the pulse. If the pulse was perceived at two sites or more, the subject was asked to report the sites and to state which site seemed most intense. Each pulse was presented 10 times, in random order.

\section{Results and Discussion}

Unifocal electrodes. The pulses were perceived at single locations or at two locations. The frequency with which the pulses were perceived at single locations (i.e., single responses) amounted to $94.5 \%$ of the total responses ( 5 pulses $\times 10$ trials $\times 13$ subjects); the frequency with which the pulses were perceived at two locations (i.e., dual responses) amounted to $5.5 \%$ of the total responses.
Thus, the pulses were mainly perceived at single locations. For the stimulation at the tip of the middle finger, the dual response rate amounted to $7.7 \%$; for the stimulation at the center of the middle finger, $5.3 \%$; for the stimulation at the base of the middle finger, $4.6 \%$; for the stimulation at the index finger, $5.4 \%$; and for the stimulation at the ring finger, $4.6 \%$. Thus it did not appear that the rate of dual responses depended on the stimulus site.

Table 1 shows the frequencies (in percent) of localization responses obtained for each of the five cathodes. For the cases in which the pulses were perceived at two locations, the site at which the more intense sensation was perceived was treated as the apparent site. No subject perceived the stimulus at the wrist or foot, so these headings are not shown in Table 1.

The rates of correct responses, which are presented in Table 1, are low for stimulation at the three sites of the middle finger, but high for the index and ring fingers. The stimulation of the middle finger was hardly ever localized on the other fingers, but was mostly localized somewhere on the middle finger. If, therefore, the subjects had selected the apparent site by pure guessing, the frequency at which each site of the middle finger was selected would have averaged $33.3 \%$, with the $95 \%$ confidence limits of $56.0 \%$ and $12.4 \%$. Thus, it is safe to say that the response site is likely to migrate proximally away from the stimulus site (the center) or is distributed equally all over the finger (the tip and the base).

On the other hand, it was very easy to localize the stimulus among the three fingers. For the stimulation of the middle finger, $99 \%$ of the total responses were obtained within the middle finger. For the index finger, most stimuli $(98 \%)$ were localized somewhere on the index finger$82 \%$ of the total responses took place at the tip of the index finger, $7 \%$ at the center, and $9 \%$ at the base. Similarly, for the ring finger, all stimuli were localized on the ring finger $-90 \%$ of the total responses took place at the tip, $5 \%$ at the center, and $5 \%$ at the base.

Concentric electrodes. Five of the subjects did not perceive a maximum pulse of $10 \mathrm{~mA}$ for the electrode placed at the center or the base of the middle finger. Therefore, the data from these subjects were not included in the analysis. The rates of perceiving the pulses at a single location or at two locations were $98.8 \%$ and $1.2 \%$ of the total re-

Table 1

Frequencies (in Percent) of the Sites Pointed by 13 Subjects with Unifocal Electrodes

\begin{tabular}{lrrrrrrr}
\hline & \multicolumn{7}{c}{ Indicated Site } \\
\cline { 2 - 7 } \multicolumn{1}{c}{ Stimulus Site } & TM & CM & BM & TI & TR & Other \\
\hline Tip of Middle (TM) & 13 & 32 & 54 & & & 1 \\
Center of Middle (CM) & 8 & 24 & 66 & 1 & 1 & \\
Base of Middle (BM) & 27 & 30 & 42 & & 1 & \\
Tip of Index (TI) & & & 1 & 82 & 1 & $16^{*}$ \\
Tip of Ring (TR) & & & & & 90 & $10 \dagger$ \\
\hline
\end{tabular}

*Localized at the center $(7 \%)$ and base $(9 \%)$ of the index finger. †Localized at the center $(5 \%)$ and base $(5 \%)$ of the ring finger. 
Table 2

Frequencies (in Percent) of the Sites Pointed by 11 Subjects with Concentric Electrodes

\begin{tabular}{lrrrrr}
\hline & \multicolumn{5}{c}{ Indicated Site } \\
\cline { 2 - 6 } \multicolumn{1}{c}{ Stimulus Site } & TM & CM & BM & TI & TR \\
\hline Tip of Middle (TM) & 95 & 1 & & & 4 \\
Center of Middle (CM) & 2 & 86 & 6 & & 6 \\
Base of Middle (BM) & & 3 & 95 & & 2 \\
Tip of Index (TI) & 1 & 1 & & 97 & 1 \\
Tip of Ring (TR) & & 1 & & 4 & 95 \\
\hline
\end{tabular}

sponses ( 5 pulses $\times 10$ trials $\times 11$ subjects), respectively. In addition, there was no systematic tendency for the perception of two locations to take place on particular fingers. Thus, for the concentric electrodes as well, the pulses were mostly perceived at single locations.

Table 2 shows the frequencies (in percent) of localization responses obtained for each of the five pairs of electrodes, in the same manner as in Table 1 . The discrimination of current among the five sites was generally very high, with correct responses of $86 \%$ or more. This is in distinct contrast with the results for the unifocal electrodes.

For each site, a Fisher's exact probability test was performed on a $2 \times 2$ contingency table, with unifocal versus concentric electrode heading the rows and correct versus error responses heading the columns for the 24 subjects. The results showed that the correct responses for the concentric electrodes were significantly higher than those for the unifocal electrodes at the tip $(p<.001)$, center $(p<$ $.01)$, and base $(p<.05)$ of the middle finger, but this was not the case for the index and ring fingers.

\section{EXPERIMENT 1B}

Table 1 indicates that the stimulation at the tip of the middle finger was localized all over the finger, but the stimulation at the tips of the index and ring fingers was localized at much the same site as the stimulus site. This suggests that there is a bias for subjects to point, not to the apparent sites of pulses, but to the electrode sites closest to the apparent sites. The purpose of Experiment $1 \mathrm{~B}$ was to reexamine the apparent sites of pulses by placing three electrodes not only on the middle finger but also on both the index and the ring fingers, and by increasing the number of finger sites to be selected by the subject as the apparent locations. It was thought that these changes in experimental setting might be useful in reducing bias, enhancing precision of measurement, and enabling us to treat the data numerically.

Single pulses were replaced by multiple pulses, since longer stimulation would make the localization judgments easier and would improve the poor localization that was found for the middle finger with the unifocal electrodes in Experiment 1A. The results of this experiment were compared with the results of Experiment $1 \mathrm{~A}$, in which single pulses were used.

\section{Method}

Subjects. Twenty-two undergraduates (21 males and 1 female) volunteered as subjects. No special qualifying criteria were imposed.

Apparatus and Electrode Placement. The appararus, electrode configuration, electrode size, electrode paste, and skin treatment were the same as those in Experiment 1A. The main stimulus parameters that were examined in Experiment 1B were the electrode site and current pulse. One unifocal cathodal electrode (or paired concentric electrodes) was (were) placed on each combination of three fingers (index, middle, and ring) at three positions (the tip, center, and base). The wrist electrode was not used in this experiment. The multiple pulses employed were 80 successive 2 -msec pulses with a stimulus onset asynchrony (SOA) of $8 \mathrm{msec}$, resulting in a train with a total duration of $634 \mathrm{msec}$.

Procedure. Ten subjects received the unifocal electrodes, and the remaining 12 subjects received the concentric electrodes. For each electrode condition, a threshold was determined at the center of the middle finger (the standard site) for each subject, in the same manner as in Experiment $1 \mathrm{~A}$.

After determination of the threshold, the pulses of 1.3-2.0 times the threshold flowed to the center of the middle finger, resulting in perceptible but nonpainful sensations. As before, the subject was then asked to equate the sensation magnitude of the standard site with the sensation magnitudes of other sites. During the determination of the threshold for the standard site and during the adjustment of the variable electrodes, the subject was not informed what the standard site was or what site was being compared with the standard site.

For the unifocal electrodes, since every subject provided a low threshold for the standard site $(M=1.30 \mathrm{~mA}, S D=.20 \mathrm{~mA})$, the suprathreshold pulses for each variable site could be determined within a range of $10 \mathrm{~mA}$. For the unifocal electrodes, 7 of the 12 subjects succeeded in determining the suprathreshold pulses, but 5 subjects did not, because the current of more than $10 \mathrm{~mA}$ was necessary to cause sensations for all sites. The mean threshold for the standard site, which was taken across the 7 subjects, was $8.36 \mathrm{~mA}(S D=1.39 \mathrm{~mA})$.

These suprathreshold pulses were delivered at one site at a time with a switch that controlled the stimulator and the electrodes. The subject was asked to report the site where the pulses were perceived. To prompt the subjects' judgments, a series of numbers from one to seven was assigned to prominent parts of each finger: (1) the extreme point, (2) the tip (the distal phalanx), (3) the second knuckle (the distal interphalangeal joint), (4) the center (the middle phalanx), (5) the first knuckle (the proximal interphalangeal joint), (6) the base (the proximal phalanx), and (7) the border between the finger and the palm. A drawing of the right hand in which the seven parts were indicated with the numbers was presented on a wall in front of the subject (see Figure 2). The subject was instructed to report the apparent sites of pulses by using these numbers. When, for example, the pulses were perceived at the first knuckle of the ring finger, he/she reported "Site 5 of the ring." When the pulses were perceived at two sites or more, the subject reported all the sites. For example, the subject reported "Sites 3-5 of the index" or "Sites 1 and 2 of the middle." In both electrode conditions, the pulse train was presented 10 times at each site, in random order.

\section{Results and Discussion}

Unifocal electrodes. The pulses that were perceived at single locations amounted to $65.0 \%$ of the total responses ( 9 sites $\times 10$ trials $\times 7$ subjects), and the pulses that were perceived at two or more locations amounted to $35.0 \%$ of the total responses. In every case in which the pulses were perceived simultaneously over several locations, the locations were within single fingers (e.g., 


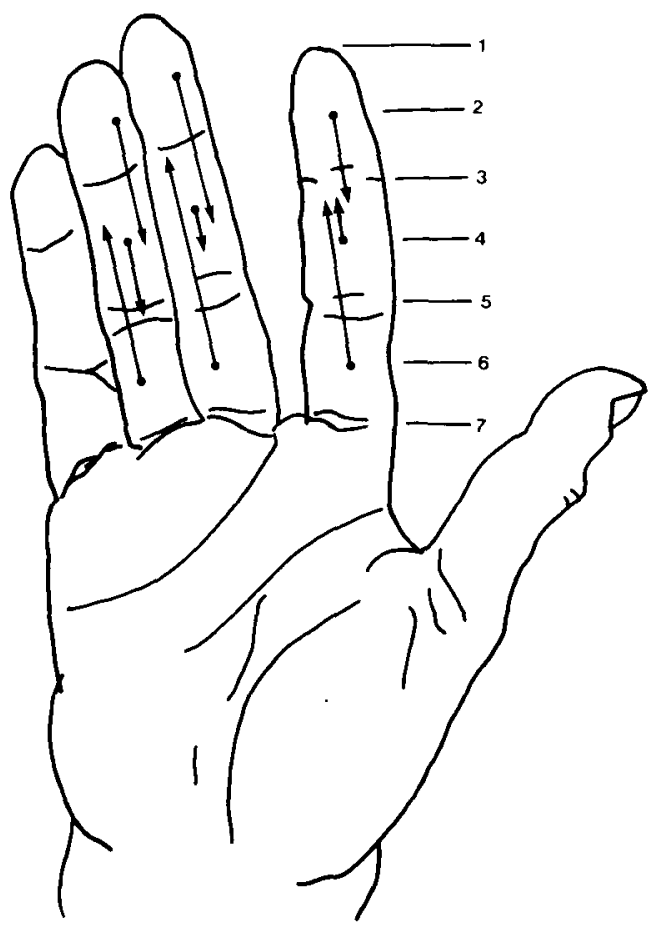

Figure 2. Mean indicated sites for the unifocal electrodes (Experiment 1B). Filled circles represent the centers of electrodes; arrowheads, the mean indicated sites. The arrows are shifted laterally to avoid overlapping. See text for the anatomical names of the finger parts in the localization task.

Sites 3,4 , and 5 of the index finger), but not over different fingers. Therefore, the center of the reported locations was treated as the apparent location.

The left half of Table 3 shows the frequencies (in percent) of localization responses obtained for each stimulated finger. It is clear that the pulses were discriminated perfectly among the three fingers.

Figure 2 shows the pointed sites that were averaged over the subjects after obtaining the mean sites for each subject. The filled circles represent the stimulus sites; the arrowheads, the mean indicated sites. A two-way (3 fingers $\times 3$ positions) repeated measures analysis of variance (ANOVA) showed that the main effect of finger was significant $[F(2,18)=3.77, p<.05]$. The stimulation of the index finger was perceived to be most distal (3.4), the stimulation of the ring finger was perceived to be most proximal (4.3), and the stimulation of the middle finger was perceived between the two (4.1).

Table 3

Frequencies (in Percent) of Finger Localization Obtained with the Unifocal (10 Subjects) and Concentric Electrodes (7 Subjects)

\begin{tabular}{lrrrrrrr} 
& \multicolumn{5}{c}{ Unifocal } & & \multicolumn{3}{c}{ Concentric } \\
\cline { 2 - 6 } \cline { 5 - 7 } Stimulus & Index & Middle & Ring & Index & Middle & Ring \\
\hline Index & 100 & 0 & 0 & 100 & 0 & 0 \\
Middle & 0 & 100 & 0 & 0 & 98 & 2 \\
Ring & 0 & 0 & 100 & 0 & 6 & 94 \\
\hline
\end{tabular}

The main effect of position was significant $[F(2,18)=$ $6.55, p<.01]$. The stimulation at the tip of the finger (2.0) was localized at its center (4.0), the stimulation at the center (4.0) was localized almost precisely (4.3), and the stimulation at the base (6.0) was localized at the region close to the second knuckle (3.4). In other words, the stimulation at the base was perceived to be most distal and the stimulation at the center was perceived to be most proximal.

The finger $\times$ position interaction was significant $[F(4,36)=4.27, p<.01]$. The test of simple main effects suggests that for the middle finger $[F(2,45)=9.74$, $p<.001]$ and the ring finger $[F(2,45)=7.88, p<$ $.001]$, the stimulation at the base was perceived to be most distal ( 3.3 for the middle and 3.7 for the ring) and the stimulation at the center was perceived to be most proximal (4.6 for the middle and 5.0 for the ring), whereas for the index finger, a significant difference did not occur $(F<1)$.

A paired-sample $t$ test was performed to compare the stimulus site and the mean indicated sites. ${ }^{3}$ For the stimulation at the tip, the mean indicated sites migrated proximally from the stimulus sites [for the index finger, $t(9)=4.39, p<.01$; for the middle finger, $t(9)=7.85$, $p<.001$; and for the ring finger, $t(9)=6.93, p<$ $.001]$. For the stimulation at the base, the mean indicated sites migrated distally from the stimulus sites [for the index finger, $t(9)=7.50, p<.001$; for the middle finger, $t(9)=7.62, p<.001$; and for the ring finger, $t(9)=$ $6.04, p<.001]$. For the stimulation at the center, the indicated site of the ring finger was significantly proximal away from the stimulus site $[t(9)=6.04, p<.001]$, and the indicated sites of the index and middle fingers were not significantly different from the stimulus sites.

It can thus be suggested that the stimulation within a finger was not discriminated at all or, even if it was discriminated, it was mislocalized in that the stimulation at the center was localized more proximally than the stimulation at the base.

Concentric electrodes. The pulses were perceived at single locations or at two or more locations. The frequency with which the pulses were perceived at single locations amounted to $87.1 \%$ of the total responses ( 9 sites $\times 10$ trials $\times 7$ subjects), and the frequency with which the pulses were perceived at two or more locations amounted to $12.9 \%$ of the total responses. When the pulses were perceived simultaneously at several locations, the centers of the reported locations were treated as the apparent sites, since there was no case in which the pulses were localized simultaneously over different fingers.

The right half of Table 3 shows frequencies (in percent) of localization responses obtained for each stimulated finger. It is clear that the pulses were discriminated almost perfectly among the three fingers.

We calculated the mean indicated sites for each subject. Excluded from this calculation were responses in which the subjects failed to point correctly to the stimulated fingers. Figure 3 shows the mean indicated sites that were taken over the subjects after obtaining the mean sites 


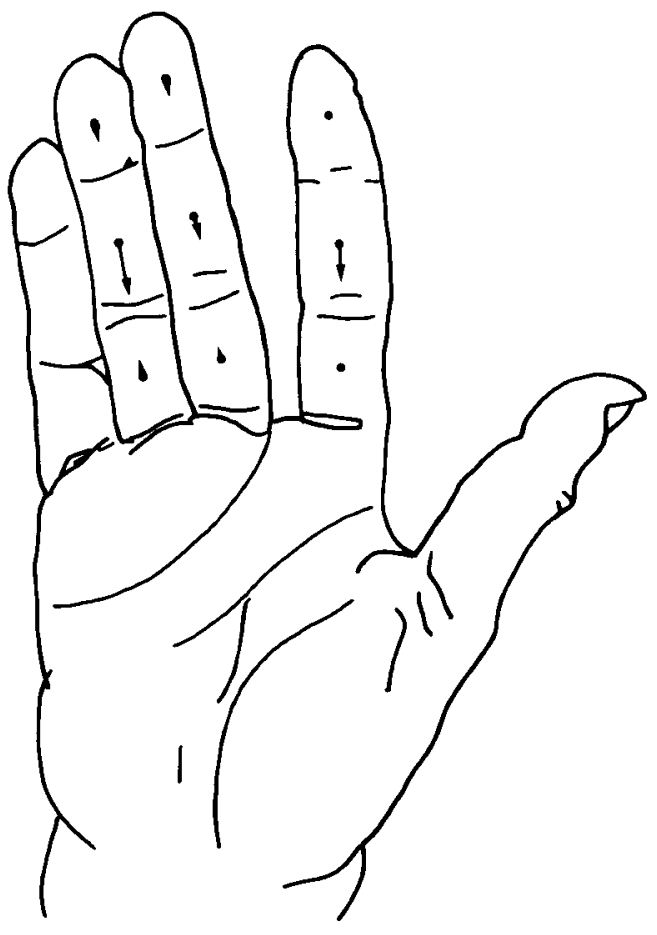

Figure 3. Mean indicated sites for the concentric electrodes (Experiment 1B). Filled circles represent the centers of electrodes; arrowheads, the mean indicated sites.

for each subject. The filled circles represent the stimulus sites; the arrowheads, the mean indicated sites. A twoway ( 3 fingers $\times 3$ positions) repeated measures ANOVA showed that the main effect of position was significant $[F(2,18)=277.2, p<.001]$. The main effect of finger and the finger $\times$ position interaction were not significant. These results mean that the pulses were discriminated within fingers as well as among fingers.

A paired-sample $t$ test was performed to compare the stimulus site and the mean indicated sites. Significant differences were obtained for the center of the index finger $[t(6)=2.74, p<.05]$ and the center of the ring finger $[t(6)=3.61, p<.05]$, but for the seven other sites, the differences were not significant. Thus it can be stated that the pulses were localized very precisely or were localized closer to the palm.

Intrasubject variability of localization. To obtain the intrasubject variability of localization, we calculated the individual standard deviations of indicated sites for each combination of configuration, finger, and position. Table 4 shows the means and the standard deviations of the intrasubject variabilities. A three-way mixed-model ANOVA, in which configuration was a between-subject factor and finger and position were within-subject factors, was performed on the intrasubject variabilities. The main effect of configuration was significant $[F(1,15)=15.72$, $p<.001]$, suggesting that the concentric electrodes $(M=$ .32) generated a smaller variability that the unifocal electrodes $(M=.65)$. The main effect of finger was sig- nificant $[F(2,30)=4.22, p<.05]$, suggesting that the variability for the ring finger (.57) was larger than that for both the index (.44) and the middle fingers (.44). The main effect of position was not significant; the interactions of configuration $\times$ finger, configuration $\times$ position, and finger $\times$ position were not significant.

Comparison between the single and multiple pulses. To examine the effects of pulse number on localization, the results of the middle finger from Experiments 1 and 2 were compared. Under the unifocal electrodes, the multiple pulses at the tip, center, and base of the middle finger were localized at $4.3,4.6$, and 3.3 , respectively, and the single pulses to the same sites were localized at 4.8, 5.2, and 4.3 , respectively. The values for the single pulses were obtained from the frequencies of the indicated sites in Table 1. For example, the mean of the indicated sites for the tip of the middle finger was obtained by $(2 \times 13+4$ $\times 32+6 \times 54) / 99$. Similarly, under the concentric electrodes, the multiple pulses at the tip, center, and base of the middle finger were localized at $2.1,4.3$, and 5.8, and the single pulses to the same sites were 2.0, 4.1, and 5.9, respectively.

Figure 4 shows the relation of indicated site between the single pulses and the multiple pulses at the middle finger. The abscissa represents the indicated site for the single pulses and the ordinate represents the indicated site

Table 4

Means and Standard Deviations of Intrasubject Variabilities for Combinations of Electrode Configuration, Finger, and Position

\begin{tabular}{|c|c|c|c|c|c|c|c|c|c|}
\hline & \multicolumn{3}{|c|}{ Index } & \multicolumn{3}{|c|}{ Middle } & \multicolumn{3}{|c|}{ Ring } \\
\hline & Tip & Center & Base & Tip & Center & Base & Tip & Center & Base \\
\hline \multicolumn{10}{|c|}{ Unifocal Electrodes } \\
\hline$M$ & .45 & .58 & .67 & .61 & .47 & .75 & .75 & .74 & .80 \\
\hline$S D$ & .26 & .24 & & .20 & .16 & .31 & .36 & .36 & .32 \\
\hline \multicolumn{10}{|c|}{ Concentric Electrodes } \\
\hline$M$ & .25 & .41 & .27 & .23 & .24 & .33 & .24 & .62 & .27 \\
\hline$S D$ & .18 & .31 & .27 & .23 & .21 & .29 & .21 & .32 & .25 \\
\hline
\end{tabular}

Note-These values were calculated from the data of categorical judgments in Experiment 1B. The means and $S D$ s in millimeters were obtained approximately, by multiplying these values by $12 \mathrm{~mm}$.

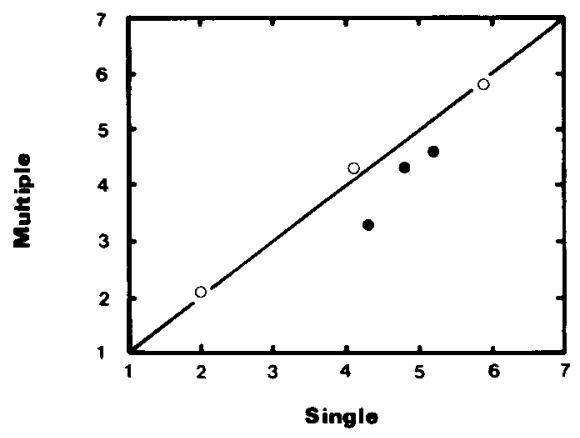

Figure 4. The relation of apparent site between the single pulses (Experiment 1A) and the multiple pulses (Experiment 1B) at the middle finger. Open circles represent the concentric electrodes; filled circles, the unifocal electrodes. 
for the multiple pulses. The parameter is the electrode configuration. The diagonal line represents a prediction obtained when the indicated sites of the two pulse conditions coincide perfectly with each other. Figure 4 suggests that despite the differences in experimental conditions, the indicated sites for the single pulses are in good agreement with those for the multiple pulses $(r=.94)$. Therefore, it does not seem that there was a prominent improvement of current localization by the multiple pulses.

\section{EXPERIMENT 1C}

To lessen the bias for the subject to point to the electrode site rather than the apparent site, one might suppose that the electrode sites should be masked from the subject's view. However, even if we had masked the stimulus sites, the bias might not have disappeared, because the subject was aware of where the electrodes were placed early in the session, and was instructed, for ethical reasons, how current was delivered to the electrodes. Experiment $1 \mathrm{C}$ was therefore designed to examine whether this bias would be reduced if the hand was concealed from the subject's view.

\section{Method}

Ten male undergraduates volunteered as the subjects. No special qualifying criteria were imposed. The multiple pulses ( 80 successive 2 -msec pulses, each with an SOA of $8 \mathrm{msec}$ ) were delivered with the unifocal electrodes that were used in Experiment 1B. The electrode position, size, paste, and skin treatment were the same as those in Experiment 1B. After determination of the threshold for the center electrode of the middle finger $(M=1.18 \mathrm{~mA}, S D=$ $.25 \mathrm{~mA}$ ), in the same manner as in Experiment $1 \mathrm{~B}$, the subject reported the apparent sites of pulses that were perceptible but not painful and that were equal to each other in subjective intensity. In the masked-hand condition, the right hand of the subject was covered with a piece of white cloth $(27 \times 27 \mathrm{~cm})$ to prevent the subject from seeing the electrode positions. The subjects in this condition judged the apparent site with reference to the drawing of the hand. In the exposed-hand condition, the masking cloth was removed so that the subject could see the hand directly. In either condition, each electrode position was randomly stimulated 10 times for each subject. Half the subjects received the masked-hand condition first and then the exposed-hand condition; the remaining subjects received the reverse order.

\section{Results and Discussion}

For the exposed-hand condition, the frequency with which the pulses were perceived at a single location amounted to $71.3 \%$ of the total responses $(9$ sites $\times 10$ trials $\times 10$ subjects), and the frequency with which the pulses were perceived at two or more locations amounted to $28.7 \%$ of the total responses. For the masked-hand condition, the frequency for a single location was $67.9 \%$ and the frequency for two or more locations was $32.1 \%$.

Table 5 shows frequencies (in percent) of localization responses obtained for each stimulated finger. Clearly, the discrimination among the three fingers was almost perfect for both viewing conditions.

A three-way ( 2 views $\times 3$ fingers $\times 3$ positions) repeated measures ANOVA was done for the mean indicated sites. The results indicated that no main effect was sig-
Table 5

Frequencies (in Percent) of Finger Localization Obtained Under the Exposed-Hand and Masked-Hand Conditions (10 Subjects)

\begin{tabular}{lrrrrrrr}
\hline & \multicolumn{5}{c}{ Response } \\
\cline { 2 - 4 } \cline { 5 - 7 } Stimulus & Index & Middle & Ring & & Index & Middle & Ring \\
\hline Index & 100 & 0 & 0 & 100 & 0 & 0 \\
Middle & 0 & 100 & 0 & 0 & 99 & 1 \\
Ring & 0 & 0 & 100 & 0 & 1 & 99 \\
\hline
\end{tabular}

nificant, and the two-way and three-way interactions were not significant. Figure 5 shows the mean indicated sites that were taken over the subjects and the viewing conditions. This means that the discrimination of pulses on a finger was very difficult in both viewing conditions.

Paired-sample $t$ tests to compare the stimulus site and the indicated site revealed significant differences for the stimulation at the tip [for the index finger, $t(19)=8.38$, $p<.001$; for the middle finger, $t(19)=9.33, p<.001$; for the ring finger, $t(19)=6.91, p<.001]$ and base [for the index finger, $t(19)=14.46, p<.001$; for the middle finger, $t(19)=6.76, p<.001$; for the ring finger, $t(19)=6.23, p<.001]$ of the fingers, but not for the stimulation at the middle of the fingers. It can therefore be suggested that, whatever the pulses stimulated on a finger, they were perceived at or near the center of the finger.

This experiment has two implications. First, it denies the possibility that the bias for the subject to be likely to

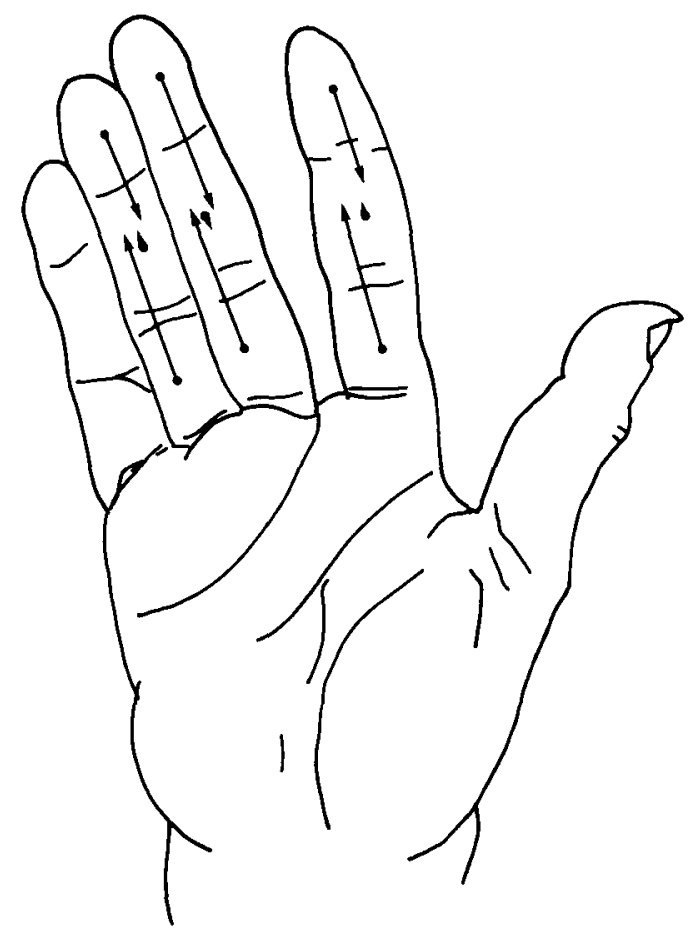

Figure 5. Mean indicated sites for the unifocal electrodes (Experiment 1C). Filled circles represent the centers of electrodes; arrowheads, the mean indicated sites. 
point to the electrode position may be reduced if the subject's hand is concealed from view. Probably the bias is triggered only if the subject recognizes the electrode positions. But even if the electrode positions are not seen directly, we may perceive them by means of cutaneous senses. The second implication is that our method of determining apparent location is very reliable, because, despite different subjects, the results in Figure 5 are very similar to those in Figure 2.

\section{EXPERIMENT 2}

An important difference between the two electrode configurations was that, for the concentric electrodes, the apparent location was likely to be judged correctly both among fingers and within a finger, whereas for the unifocal electrodes, it was judged correctly among fingers but not within a finger. Another difference is that the mean threshold for the concentric electrodes was four times or more as high as that for the unifocal electrodes. One possible explanation of these differences is that the concentric electrodes limit current to a small superficial region, but the unifocal electrodes probably generate many stray current paths along the longitudinal axis of the forearm. For example, the cathode placed on the tip of the middle finger depolarizes not only the nerves just beneath the cathode, but other nerves under other sites on the same finger. As a result, current may be perceived all over the middle finger.

Experiment 2 was a repetition of Experiment 1B, but with the forearm rather than the fingers. The stimuli were the multiple pulses used in Experiment 1B. If the unifocal electrodes generated stray current paths that might disturb the localization of current, the concentric electrodes would also provide better localization in Experiment 2.

\section{Method}

Apparatus and electrode placement. The apparatus and electrodes were the same as those used in Experiment 1A. For the unifocal electrodes, an anodal electrode $30 \mathrm{~mm}$ in diameter was placed on the instep of the right foot, and seven cathodal electrodes, each of which was $7 \mathrm{~mm}$ in diameter, were placed longitudinally or transversely on the right forearm as shown in Figure 6. For the concentric electrodes, seven pairs of concentric electrodes were placed at the sites shown in Figure 6. The cathodal electrode (or paired concentric electrodes) on the forearm was (were) separated by a center-to-center distance of $26 \mathrm{~mm}$ from the adjacent electrode(s).

Each unifocal electrode was filled with keratin paste and was attached to the skin, which was first washed with an alcohol solution. The concentric electrodes were placed directly on the skin, which was first washed with an alcohol solution and was rubbed with SkinPure gel. For both configurations, each electrode was fixed with a piece of semitransparent surgical tape, on which a letter was written, as in Figure 6. Electrodes B, D, and F were activated, and Electrodes A, C, E, and $\mathrm{G}$ were dummy electrodes that were never activated during the session.

Subjects. For the unifocal electrodes, 10 male undergraduates served as subjects in each of the longitudinal and transverse conditions, and 6 of these subjects took part in both conditions. Thus there were 4 subjects in the longitudinal condition only and 4 in the transverse condition only. The longitudinal condition was followed by the transverse condition 2 months later. For the concentric electrodes, 24 undergraduates served as subjects. Twelve (10 males
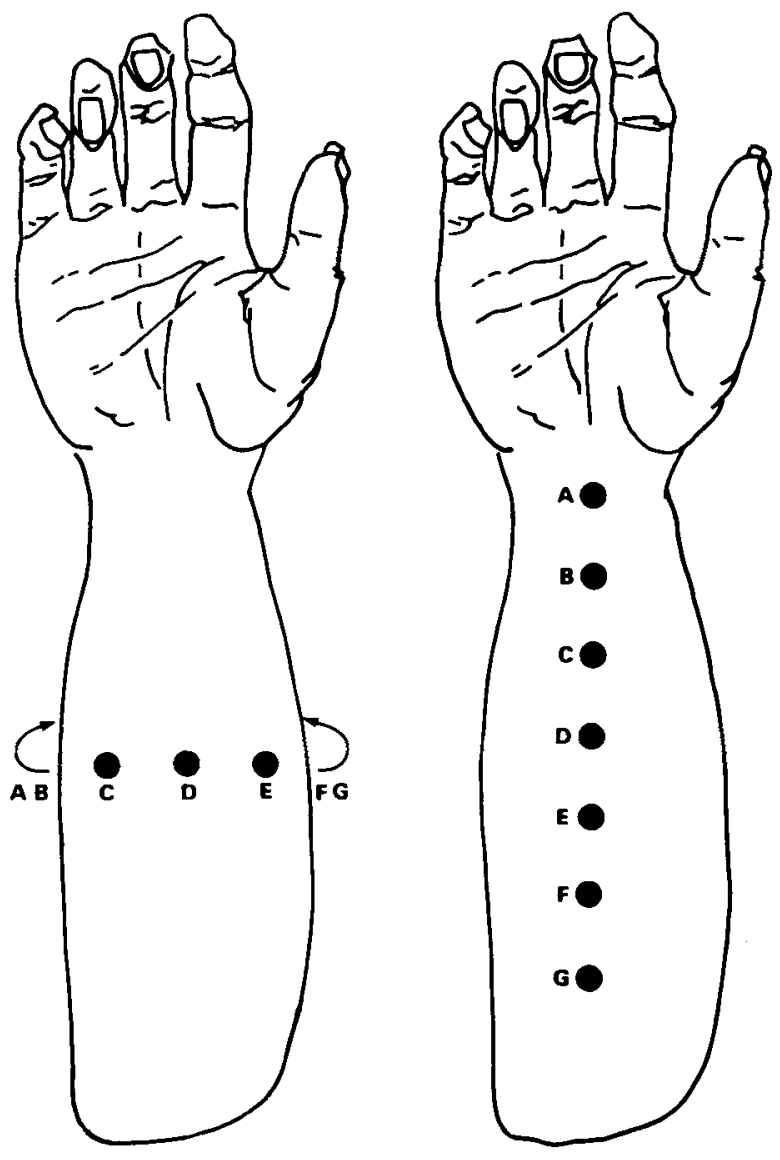

Figure 6. Seven electrodes placed transversely (left) and longitudinally (right) on the forearm. The center-to-center distance between two adjacent electrodes is $26 \mathrm{~mm}$.

and 2 females) participated in the longitudinal condition; the remaining 12 ( 11 males and 1 female) were in the transverse condition.

Procedure. For each subject, the multiple pulses used in Experiment IB were delivered to Site D, and an absolute threshold was determined with a staircase procedure, as in Experiment 1A. The subject did not know what electrodes were activated while the threshold was being determined. The mean threshold for the concentric electrodes $(M=1.69 \mathrm{~mA}, S D=.51 \mathrm{~mA})$ was significantly higher than that for the unifocal electrodes $(M=.62 \mathrm{~mA}, S D=.21 \mathrm{~mA})$ $[t(36)=4.25, p<.001]$. For the unifocal electrodes, 8 of the 14 subjects each provided a threshold and the remaining subjects each provided two thresholds over an interval of 2 months. For the latter subjects, the mean of two thresholds was taken as an individual threshold.

After determination of a threshold for Site D, the multiple pulses of 1.3-2.0 times the threshold current flowed separately to the three sites $B, D$, and F. According to the direction of the subject, the experimenter adjusted the current for each electrode, so that the pulses at the three electrodes were perceived easily as being equal but were neither unpleasant nor painful. When the subjects perceived nothing or a very faint touch, the current was raised; when the current felt painful or unpleasant, it was lowered. Through this successive comparison, a suprathreshold current was determined for each electrode. No current was delivered to Site A, C, E, or $G$. During the successive comparison, the subject was not told which electrodes were activated or which were the dummy electrodes. According to the interviews with the subjects after the session, they thought that all the electrodes had been activated. 
These suprathreshold multiple pulses were delivered to one site at a time $(B, D$, or $F)$ with a switch that controlled the stimulator and the electrodes. The subject was asked to report the site where the pulses were perceived, while viewing the forearm. When the pulses were perceived to be under an electrode at the forearm, the subject reported the letter of that electrode. When the puises were perceived to be somewhere midway between two adjacent electrodes, the subject reported the letters of both electrodes. When the pulses were perceived to be somewhere outside the end electrodes, the subject pointed to that place with the left index finger, without touching the skin. In this case, the experimenter measured the distance of the indicated site from the end electrode, with the accuracy of $1 \mathrm{~mm}$. When the pulses were perceived at two sites or more, the subject pointed to the sites and reported which site was perceived as being most intense. Each pulse train was presented 10 times in random order.

\section{Results and Discussion}

Unifocal electrodes. Most pulses were perceived at single locations. For the longitudinal condition, the rates of perceiving pulses at single and two locations were $98.0 \%$ and $2.0 \%$ of the total responses ( 10 subjects $\times 3$ cathodes $\times 10$ trials), respectively. For the transverse condition, all the stimuli were perceived at single locations. Therefore only the single-localization responses were analyzed.

The sites pointed to by each subject were transformed into distances from Site D. In this transformation, it was arbitrarily decided that for the longitudinal condition, the elbow (proximal) side of Site D was considered positive, and the wrist (distal) side of Site D, negative; for the transverse condition, the thumb (right) side of Site D was considered positive, and the little finger (left) side of Site D, negative.

Figure 7 shows the mean indicated sites that were superimposed on the stimulus sites. The filled circles rep-

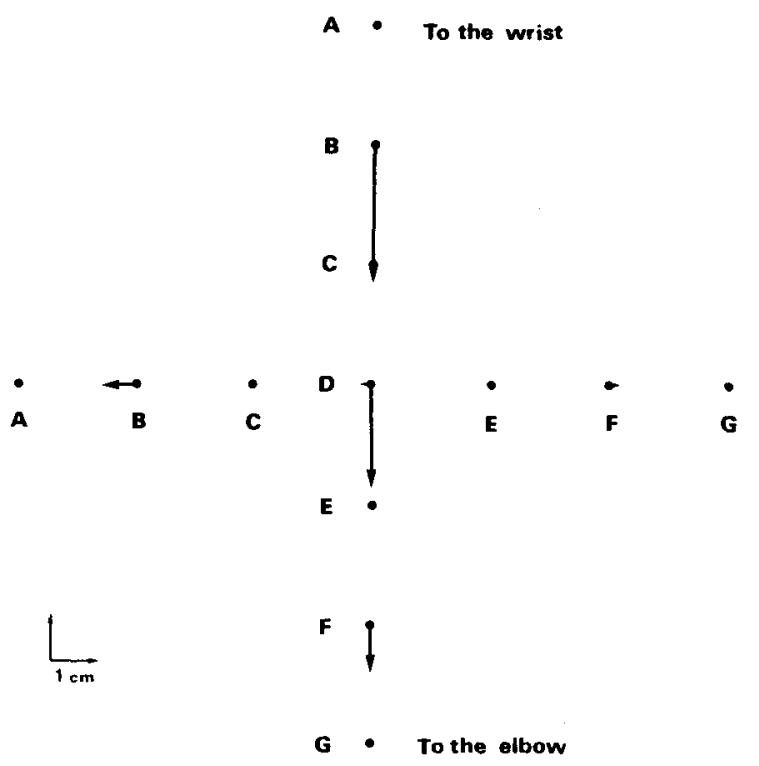

Figure 7. Mean indicated sites for the unifocal electrodes. Filled circles represent the centers of electrodes; arrowheads, the mean indicated sites. Electrodes $B, D$, and $F$ are stimulus electrodes, and Electrodes A, C, E, and G are dummy electrodes. A scale of $1 \mathrm{~cm}$ is given at the bottom left.
Table 6

Means and Standard Deviations of Intrasubject Variabilities (in Millimeters) for Combination of Electrode Configuration, Body Axis, and Activated Site

\begin{tabular}{lrrrrrrr}
\hline & \multicolumn{3}{c}{ Longitudinal } & & \multicolumn{3}{c}{ Transverse } \\
\cline { 2 - 4 } \cline { 5 - 7 } & $\mathrm{B}$ & $\mathrm{D}$ & $\mathrm{F}$ & $\mathrm{B}$ & $\mathrm{D}$ & $\mathrm{F}$ \\
\hline \multicolumn{8}{c}{ Unifocal Electrodes } \\
$S D$ & 20.6 & 21.4 & 16.6 & 10.1 & 9.6 & 7.7 \\
\multicolumn{8}{c}{ Concentric Electrodes } \\
$M$ & 8.3 & 6.4 & 10.0 & 7.1 & 4.2 & 4.1 \\
$S D$ & 21.1 & 21.5 & 12.6 & 10.4 & 13.3 & 10.4 \\
& 4.5 & 8.4 & 5.4 & 2.3 & 3.1 & 4.3 \\
\hline
\end{tabular}

resent the stimulus sites and the arrowheads represent the mean indicated sites. A one-way repeated measures ANOVA was performed on the distances obtained for each axis. The results showed that the main effect of stimulus site was significant for the longitudinal axis $[F(2,18)=53.8, p<.001]$ and the transverse axis $[F(2,18)=307.7, p<.001]$. This suggests that the three stimulus sites were readily discriminated for both axes.

However, the mean distances of the indicated sites from the stimulus site in the longitudinal condition were significant (for Site B, $t(9)=4.13, p<.01$; for Site D, $t(9)=2.88, p<.05$; and for Site $\mathrm{F}, t(9)=3.25, p<$ $.01]$. On the other hand, the mean distances of the indicated sites from the stimulus site in the transverse condition were not significant. Thus, the pulses in the longitudinal condition were localized closer to the elbow (or proximally), but the pulses in the transverse condition were localized in accordance with stimulus site.

To determine intrasubject variability, we calculated individual standard deviations of the distances obtained for each combination of body axis and stimulus site. The upper half of Table 6 shows the means and the standard deviations of the individual intrasubject variabilities. For the 6 subjects who participated in both axis conditions, a twoway ( 2 axes $\times 3$ sites) repeated measures ANOVA was performed, showing that the main effect of axis was significant $[F(1,5)=67.08, p<.001]$, but the main effect of site and the axis $\times$ site interaction were not significant. For the 8 subjects who participated only in one axis condition, a two-way ( 2 axes $\times 3$ sites) mixed-model ANOVA showed that the main effect of axis was significant $[F(1,6)=19.18, p<.01]$, whereas the main effect of site and the axis $\times$ site interaction were not. A one-way repeated measures ANOVA for each body axis showed that the main effect of site was not significant for both axis conditions. Thus, the intrasubject variability was larger for the longitudinal axis than for the transverse axis, but it did not differ among the sites along each axis.

Concentric electrodes. For the concentric electrodes, all the pulses were perceived at single locations. The sites indicated by each subject were transformed in terms of distances from Site D, in the same manner as we did for the unifocal electrodes. Figure 8 shows the mean indicated sites that were superimposed on stimulus sites. The filled circles represent the stimulus sites and the arrow- 


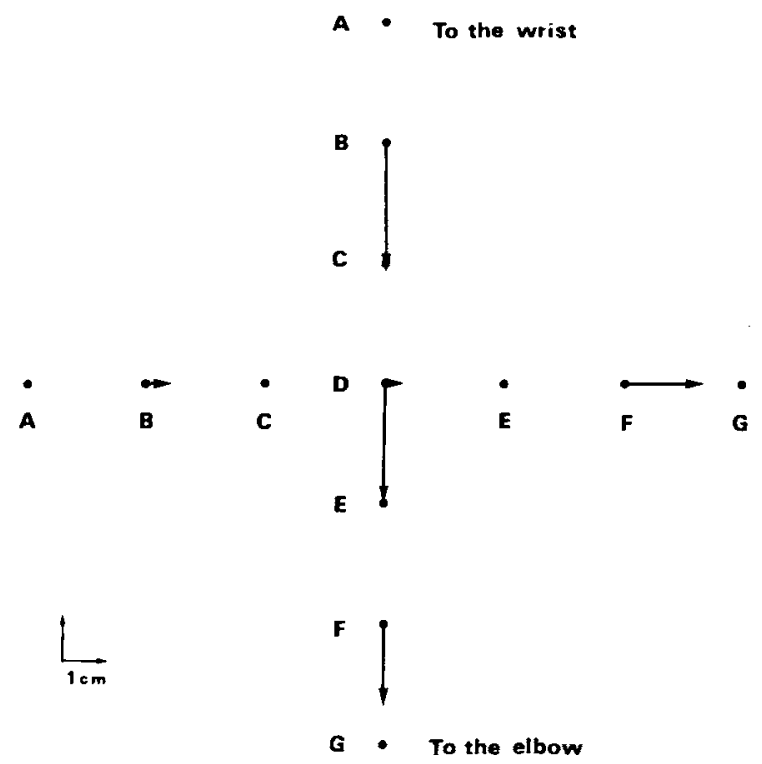

Figure 8. Mean indicated sites for the concentric electrodes. Filled circles represent the centers of electrodes; arrowheads, the mean indicated sites. Electrodes $\mathrm{B}, \mathrm{D}$, and $\mathrm{F}$ are stimulus electrodes, and Electrodes A, C, E, and $G$ are dummy electrodes. A scale of $1 \mathrm{~cm}$ is given at the bottom left.

heads represent the mean indicated sites. A one-way repeated measures ANOVA was performed on the distances obtained along each axis. The results show that main effect of site was significant for the longitudinal axis $[F(2,22)=148.6, p<.001]$ and the transverse axis $[F(2,22)=571.1, p<.001]$. Thus, it is evident that the three sites were discriminated along both axes.

In the longitudinal condition, the mean distances from the stimulus site were significant from the stimulus site (for Site B, $t(11)=4.68, p<.001$; for Site D, $t(11)=$ $4.24, p<.01$; and for Site F, $t(11)=9.85, p<.001$ ] For the transverse condition, only the mean distance of Site $\mathrm{F}$ from the stimulus site was significant $[t(11)=$ $2.28, p<.05$ ]. Thus, the pulses for the longitudinal condition were localized proximally away from the stimulus site, whereas the pulses for the transverse condition were localized at much the same place as the stimulus site.

The lower half of Table 6 shows the means and the standard deviations of the individual intrasubject variabilities obtained for combinations of body axis and stimulus site. A two-way ( 2 axes $\times 3$ sites) mixed-model ANOVA was performed on the standard deviations. The results showed that the main effect of axis was significant $[F(1,22)=$ $30.84, p<.001]$ and that the main effect of site was significant $[F(2,44)=7.99, p<.01]$. The axis $\times$ site interaction was also significant $[F(2,44)=4.11, p<.05]$. The simple main effect of site was significant under the longitudinal condition $[F(2,44)=75.37, p<.001]$, but not under the transverse condition. This suggests that for the longitudinal condition, Site F provided a smaller standard deviation than did Sites B and D, whereas for the transverse condition, the standard deviation did not differ among the sites.

\section{GENERAL DISCUSSION}

\section{Transverse Placement}

The most reliable finding obtained in this study is that the transverse placement of electrodes at the fingers and forearm caused localization at much the same place as the stimulus site. For the fingers, the subjects correctly identified the stimulated fingers with $94 \%$ or greater accuracy. For the forearm, the errors of localization were, on the average, $4.1 \mathrm{~mm}$ for the unifocal electrodes and $5.0 \mathrm{~mm}$ for the concentric electrodes. In addition, the intrasubject variability of localization judgments at the forearm was smaller for the transverse placement of electrodes $(10 \mathrm{~mm})$ than for their longitudinal placement $(19 \mathrm{~mm})$. These results parallel those of Blamey and Clark (1987), who reported that, when eight unifocal electrodes stimulated the digital nerve bundles of the index, middle, ring, and little fingers (i.e., two electrodes for each finger), all subjects identified the stimulus site with $95 \%$ or greater accuracy.

\section{Longitudinal Placement}

Forearm. The localization of the longitudinal placement of electrodes varied in a somewhat complicated way, as a function of electrode configuration (unifocal vs. concentric) and body site (fingers vs. forearm). At the forearm, the results obtained with the two configurations were similar. On the whole, the longitudinal placement of electrodes was likely to cause localization on the proximal side of the stimulus site. The mean error of localization was $21.2 \mathrm{~mm}$ for the unifocal electrodes and $22.5 \mathrm{~mm}$ for the concentric electrodes. In addition, the intrasubject variability did not differ between the unifocal (14.3-mm) and the concentric (14.9-mm) electrodes. These findings suggest that the stray current that may be produced by the unifocal electrodes did not affect the localization judgments.

The error of localization was smaller for the stimulus site closer to the elbow. For the unifocal electrodes, the distances between the indicated sites and stimulus site were $29.9,23.3$, and $10.4 \mathrm{~mm}$ for Sites B, D, and F, respectively, and for the concentric electrodes, they were 27.1, 25.9 , and $14.4 \mathrm{~mm}$ for Sites B, D, and F, respectively. Although there seems to be no persuasive explanation for this mislocalization, it may be explained by the anchor theory, which assumes that the localization of a stimulus on the skin tends to migrate toward the anchoring region (Boring, 1942). The wrist, elbow, and knuckle have often been considered as anchors. If the elbow acted as an anchor in our study, point stimuli on the forearm were localized toward the elbow. However, this theory does not explain why the wrist did not seem to act as an anchor. If the wrist were an anchor, point stimuli close to the wrist (e.g., Site B) would have been localized toward the wrist. 
Our results appear to predict the apparent distance between two points on the forearm. If the apparent distance equals the difference between the two indicated sites, the mean apparent distances of BD and DF are 48.1 and $39.8 \mathrm{~mm}$, respectively. Likewise, the mean apparent distance of BF would be $87.9 \mathrm{~mm}$. These values are smaller than the actual distances $(52 \mathrm{~mm}$ for both $\mathrm{BD}$ and $\mathrm{DF}$, and $104 \mathrm{~mm}$ for BF). Thus, the longitudinal distance is perceived to be diminished, in comparison with the transverse distance, which is processed nearly veridically. This prediction seems to be supported by direct estimation of apparent distance for electrocutaneous stimuli (Marks, Girvin, Quest, et al., 1982) and tactile pressures (Green, 1982).

Fingers. For the stimulation to the fingers, the concentric electrodes provided very precise localization or caused localization slightly proximally to the stimulus site. If the size of each response category used in Experiments 1B and $1 \mathrm{C}$ is assumed to have been $12 \mathrm{~mm}$, the mean error of localization shown in Figure 3 was $2.7 \mathrm{~mm}$, with a range of 0.5-7.1 mm, which was smaller than that obtained at the forearm. On the other hand, the unifocal electrodes produced greater errors of localization at the tip and base of the fingers. As shown in Figures 2 and 5, the stimulation at the tip of the fingers produced an error of $22 \mathrm{~mm}$ proximally; the stimulation at the base, an error of $29 \mathrm{~mm}$ distally; and the stimulation at the center, an error of $1.4 \mathrm{~mm}$ proximally. Furthermore, the intrasubject variability for the unifocal electrodes was about twice as large as that for the concentric electrodes (Table 4). These findings suggest that the stray current disturbs the localization for the unifocal electrodes.

The two configurations caused different localizations on the fingers, but not on the forearm. This interaction of site and configuration can be explained tentatively by means of the fact that the neural network is much more condensed and complicated in the fingers than in the forearm. In the fingers, the concentric electrodes stimulate the limited superficial nerves, whereas the unifocal electrodes stimulate not only the superficial nerves, but also the nerves that lie in deeper tissue. If many different nerves in a finger are stimulated simultaneously, we may be likely to judge the apparent location to be at the center of the finger (i.e., a central tendency in judgments). On the other hand, either configuration may stimulate the same superficial cutaneous nerves at the forearm, because, since the neural density at the forearm is low, even the current delivered with the unifocal electrodes may not reach deeper nerves.

\section{Comparison With Other Cutaneous Modalities}

Error direction. Because the concentric electrodes may limit current pulses to a small, definite region, it seems that the localization obtained with the use of the concentric electrodes should be compared with the localization obtained through other cutaneous modalities. The finding that localization with longitudinal placement of the electrodes on the forearm was more proximal than the stimulus site appears to resemble the results of previous studies. Studying the localization of chemical and tactile stimuli on the forearm, Green and Flammer (1989) found that tactile localization exhibited a consistent bias toward the elbow, whereas the chemical stimulus was mislocalized toward the wrist early in the session and toward the elbow late in the session. Likewise, Taus, Stevens, and Marks (1975) found that when two sites on the forearm were stimulated by radiant heat, there was a slight tendency for warmth to be localized at the proximal site. Culver (1970), who studied the localization of tactile stimuli on the palm, found that apparent location migrated toward the thumb and wrist.

Our finding may run counter to some observations, however: Parrish (1897) and Pillsbury (1895) showed that tactile stimulation on the forearm near the wrist was localized toward the wrist, and Dimmick (1915) demonstrated that radiant heat applied to the forearm was localized at the center of a given region to which the stimuli could be applied.

Error size. The error of localization of current is remarkably smaller on fingers $(2.7 \mathrm{~mm})$ than it is on the forearm $(13.8 \mathrm{~mm})$. This difference between the two sites may have been obtained for tactile pressure: Weinstein (1968) showed that the mean error of localization (the point localization threshold in his terminology) for tactile pressure was about $2 \mathrm{~mm}$ on the fingers and about $10 \mathrm{~mm}$ on the forearm.

The error of localization for the longitudinal placement of electrodes $(22.5 \mathrm{~mm})$ was remarkably larger than that for the transverse placement $(5.0 \mathrm{~mm})$. This strong anisotropic tendency was also found for radiant heat: Dimmick (1915, Table 1) demonstrated that the longitudinal error of localization on the forearm was $29.6 \mathrm{~mm}$ on the average and the transverse error was $10.5 \mathrm{~mm}$.

The mean error of localization on the forearm $(13.8 \mathrm{~mm})$ seems to be equal to or somewhat larger than that for tactile stimuli. Green and Flammer (1989) obtained an average error of $14 \mathrm{~mm}$; Weinstein (1968) obtained about $10 \mathrm{~mm}$; Weber, Kottenkamp, and Ullrich, whose data are cited in Boring (1942, p. 485), obtained 4.4-8.7 mm. However, the variability of localization of current may not be as great as that for thermal and chemical stimuli. Dimmick (1915) compared localization of radiant heat and tactile pressure on the forearm and found that the error of localization of heat was two or three times as great as that of pressure. Pritchard (1931) found that the error of localization of warmth was twice as great as that of touch. Green and Flammer (1989) found that the error of localization of chemical stimulation was 1.2-2.4 times as great as that of tactile pressure. Thus, the error size for current may be similar to that for tactile pressure, rather than radiant heat and chemical stimuli.

\section{REFERENCES}

Anderson, A. B., \& Munson, W. A. (1951). Electrical excitation of nerves in the skin at audiofrequencies. Journal of the Acoustical Society of America, 23, 155-159. 
BEKÉSY, G. von. (1959a). Neural funneling along the skin and between the inner and outer hair cells of the cochlea. Journal of the Acoustical Society of America, 31, 1236-1249.

BÉKÉSY, G. VON. (1959b). Synchronism of neural discharges and their demultiplication in pitch perception on the skin and in hearing. Journal of the Acoustical Society of America, 31, 338-349.

Békésy, G. von. (1967). Sensory inhibition. Princeton, NJ: Princeton University Press.

Blamey, P. J., \& Clark, G. M. (1987). Psychophysical studies relevant to the design of a digital electrotactile speech processor. Journal of the Acoustical Society of America, 82, 116-125.

BorING, E. G. (1930). The two-point limen and the error of localization. American Journal of Psychology, 42, 446-449.

BorING, E. G. (1942). Sensation and perception in the history of experimental psychology. New York: Appleton-Century-Crofts.

Cholewiak, R. W., \& Collins, A. A. (1991). Sensory and physiological bases of touch. In M. A. Heller \& W. Schiff (Eds.), The psychology of touch (pp. 23-60). Hillsdale, NJ: Erlbaum.

Culver, C. M. (1970). Errors in tactile localization. American Journal of Psychology, 83, 420-427.

Dimmick, F. L. (1915). On the localization of pure warmth sensations. American Journal of Psychology, 26, 142-150.

GiBson, R. H. (1968). Electrical stimulation of pain and touch. In D. R. Kenshalo (Ed.), The skin senses (pp. 223-261). Springfield, IL: Thomas.

GILMER, B. H. vON (1961). Toward cutaneous electropulse communication. Journal of Psychology, 52, 211-222.

Girvin, J. P., Marks, L. E., Antunes, J. L., Quest, D. O., OKKeefe, M. D., Ning, P., \& Dobelle, W. H. (1982). Electrocutaneous stimulation $I$. The effects of stimulus parameters on absolute threshold. Perception \& Psychophysics, 32, 524-528.

GreEN, B. G. (1982). The perception of distance and location for dual tactile pressures. Perception \& Psychophysics, 31, 315-323.

Green, B. G., \& Flammer, L. J. (1989). Localization of chemical stimulation: Capsaicin on hairy skin. Somatosensory \& Motor Research, 6, 553-566.

Higashiy ama, A. (in press). Electrocutaneous spatial integration at suprathreshold levels: An additive neural model. Joumal of Experimental Psychology: Human Perception \& Performance.

Higashiyama, A., \& Rollman, G. B. (1991). Perceived locus and intensity of electrocutaneous stimulation. IEEE Transactions on Biomedical Engineering, 38, 679-686.

Higashiyama, A., \& Tashiro, T. (1983). Temporal and spatial integration for electrocutaneous stimulation. Perception \& Psychophysics, 33, 437-442.

HigashiYama, A., \& TAShiro, T. (1988). Temporal integration of double electrical pulses. Perception \& Psychophysics, 43, 172-178.

Higashiyama, A., \& Tashiro, T. (1990). Electrocutaneous spatial integration at threshold: The effects of electrode size. Perception \& Psychophysics, 48, 389-397.

JONES, F. N. (1956). Space-time relationship in somesthetic localization. Science, 124, 484.

KIRMAN, J. H. (1974). Tactile apparent movement: The effects of interstimulus onset interval and stimulus duration. Perception \& Psychophysics, 15, 1-6.

KIRMAN, J. H. (1975). The effect of number of stimulators on the optimal interstimulus onset interval in tactile apparent movement. Perception \& Psychophysics, 17, 263-267.

Marks, L. E., Girvin, J. P., O'Keefe, M. D., Ning, P., Quest, D. O., Antunes, J. L., \& Dobelle, W. H. (1982). Electrocutaneous stimulation III. The perception of temporal order. Perception \& Psychophysics, 32, 537-541.

Marks, L. E., Girvin, J. P., Quest, D. O., Antunes, J. L., Ning, P., O'KeEFe, M. D., \& Dobelle, W. H. (1982). Electrocutaneous stimulation II. The estimation of distance between two points. Perception \& Psychophysics, 32, 529-536.

PARRsh, C. S. (1897). Localization of cutaneous impression by arm movement without pressure upon the skin. American Joumal of Psychology, 8, 250-267.

Pillsbury, W. B. (1895). Some questions of the cutaneous sensibility. American Journal of Psychology, 7, 42-57.

Prior, R. E. (1972). Study of electrocutaneous parameters relevant to dynamic tactual communication systems. Dissertation Abstracts International, 33/01-B, p. 167 (University Microfilms No. 72-20, 469).

Pritchard, E. A. B. (1931). Cutaneous tactile localization. Brain, 54, 350-371.

SAUNDERS, F. A. (1973). Electrocutaneous displays. In F. A. Geldard (Ed.) Cutaneous communication systems and devices (pp. 20-26). Austin, TX: Psychonomic Society.

Sherrick, C. E., \& Cholewiak, R. W. (1986). Cutaneous sensitivity. In K. R. Boff, L. Kaufman, \& J. P. Thomas (Eds.), Handbook of perception and human performance: Vol. I. Sensory processes and perception (chap. 12). New York: Wiley.

Sherrick, C. E., \& Rogers, R. (1966). Apparent haptic movement. Perception \& Psychophysics, 1, 175-180.

Solomonow, M., \& LYMAN, J. (1980). Electrotactile stimulation relevant to sensory-motor rehabilitation: A progress report. Bulletin of Prosthetics Research, 17, 63-72.

Solomonow, M., Lyman, J., \& Freedy, A. (1977). Electrotactile twopoint discrimination as a function of frequency, body site, laterality, and stimulation codes. Annals of Biomedical Engineering, 5, 47-60.

SPARKS, D. W. (1979). The identification of the direction of electrocutaneous stimulation along lineal multistimulator arrays. Perception \& Psychophysics, 25, 80-87

Taus, R. H., Stevens, J. C., \& Marks, L. E. (1975). Spatial localization of warmth. Perception \& Psychophysics, 17, 194-196.

TURSKY, B. (1973). Physical, physiological, and psychological factors that affect pain reaction to electric shock. Psychophysiology, 11, 95-112.

TURSKY, B., \& O'CONNELL, D. (1972). Reliability and interjudgment predictability of subjective judgments of electrocutaneous stimulation. Psychophysiology, 9, 290-295.

TURSKY, B., WATSON, P. D., \& O'CONNELL, D. (1965). A concentric shock electrode for pain stimulation. Psychophysiology, 1, 296-298.

Weber, E. H. (1978). The sense of touch (H. E. Ross \& D. J. Murray, trans.) London: Academic Press. (Original work published in 1834) WEINSTEIN, S. (1968). Intensive and extensive aspects of tactile sensitivity as a function of body part, sex, and laterality. In D. R. Kenshalo (Ed.), The skin senses (pp. 195-222), Springfield, IL: Thomas.

WIELAND, B. A. (1960). The interaction of space and time in cutaneous perception. American Journal of Psychology, 73, 248-255.

\section{NOTES}

1. In several previous studies, the assignment of polarity to the con centric electrodes was opposite to that used in this study. The central electrode was the anode, and the surrounding electrode was the cathode. Although the use of a smaller electrode as the cathode may yield more frequent reports of pain (Prior, 1972), we used perceptible but nonpainful pulses, so that the assignment of polarity might not result in a serious problem in the localization task. Note too that the assignment of polarity may not be important in the determination of the threshold for the concentric electrodes. In Experiment A in the Appendix, we compared the thresholds between the condition in which the cathode was assigned to the inner disk and the condition in which it was assigned to the surrounding ring, but a significant difference was not obtained.

2. One may suppose that, since the cathode diameter of the concentric electrodes was half the cathode diameter of the unifocal electrodes, the concentric electrodes enhance the spatial sharpness of sensation, in comparison with the unifocal electrodes. Although we did not ask the subjects about the spatial sharpness directly, according to their spontaneous reports for the threshold pulses, the concentric electrodes may cause a superficial sensation like the faint touch of a feather, and the unifocal electrodes may induce a steady tap sensation as if the skin were pulled or were flicked by nails. On the basis of the verbal reports alone, however, it seems difficult to decide what electrodes provide spatially sharper sensations.

3. The $t$ test was performed independently of an ANOVA, not post hoc.

\section{APPENDIX}

In this study, we used keratin paste for the unifocal electrodes and did not use any paste for the concentric electrodes, except 
Table A1

Means and Standard Deviations of Intrasubject Variabilities (in Millimeters) for Two Electrode Configurations

\begin{tabular}{|c|c|c|c|c|c|c|c|}
\hline \multicolumn{5}{|c|}{ Concentric } & & & \\
\hline & \multicolumn{2}{|c|}{ Large } & \multicolumn{2}{|c|}{ Small } & \multicolumn{3}{|c|}{ Unifocal } \\
\hline & $\mathrm{C}+/ \mathrm{S}-*$ & $\mathrm{C}-/ \mathrm{S}++$ & $\mathrm{C}+/ \mathrm{S}-$ & $\mathrm{C}-/ \mathrm{S}+$ & 2.5 & 5.0 & 10 \\
\hline$M$ & 1.85 & 1.70 & 3.43 & 3.54 & 2.73 & 2.01 & 1.29 \\
\hline$S D$ & 0.74 & 0.80 & 1.45 & 1.61 & 1.07 & 0.73 & 0.34 \\
\hline
\end{tabular}

Note-For the concentric electrodes, there were four combinations of size and polarity; for the unifocal electrodes, there were three cathodal sizes. *The center disk is the anode and the surrounding ring is the cathode. †The center disk is the cathode and the surrounding ring is the anode.

for rubbing the skin with Nihonkoden SkinPure gel. The threshold differences obtained in Experiments 1 and 2 might, therefore, reflect the differences of the skin treatments, rather than the electrode configuration. Similarly, the assignment of polarity for the concentric electrodes might affect the current threshold. The purpose of these supplemental experiments was, therefore, to examine the effects of keratin paste, SkinPure gel, electrode size, and polarity on the threshold.

\section{Experiment A}

In Experiment $\mathrm{A}$, we examined how the electrode configuration and polarity affect the current threshold under the same skin treatment. There were three pairs of unifocal electrodes and two pairs of concentric electrodes. For the unifocal electrodes, the cathode, which was placed on the ventral side of the right forearm, was $2.5 \mathrm{~mm}\left(4.9 \mathrm{~mm}^{2}\right), 5.0 \mathrm{~mm}\left(19.6 \mathrm{~mm}^{2}\right)$, or $10 \mathrm{~mm}$ in diameter $\left(78.5 \mathrm{~mm}^{2}\right)$, and the anode, which was placed on the instep of the right foot, was $30 \mathrm{~mm}$ in diameter. The two pairs of concentric electrodes were placed on the ventral side of the right forearm: The small one was the same as that used in Experiment 1A; the large one consisted of an inner disk $10 \mathrm{~mm}$ in diameter $\left(78.5 \mathrm{~mm}^{2}\right)$ and an outer ring of $24-\mathrm{mm}$ outside diameter and $15-\mathrm{mm}$ inside diameter $\left(275.5 \mathrm{~mm}^{2}\right)$. For the concentric electrodes, the inner disk was cathodal and the outer ring was anodal, and vice versa. Thus, there were four conditions for the concentric electrodes and three conditions for the unifocal electrodes. The threshold for single pulses of 2-msec duration was determined for each condition in the same manner as in Experiment 1A. Ten undergraduates volunteered as subjects. The forearm and instep of the subject were washed with an alcohol solution and were rubbed with Nihonkoden SkinPure gel. No paste was used for either unifocal or concentric electrodes.

Table A1 shows the mean threshold for each of the seven conditions. A two-way repeated measures ANOVA, done for the concentric electrodes, showed that the threshold was significantly smaller for the large pair than for the small pair $[F(1,9)=24.0$, $p<.001]$. But the main effect of polarity and the polarity $\times$ size interaction were not significant. A one-way repeated measures ANOVA, done for the unifocal electrodes, showed that a small threshold was obtained with a large cathode $[F(2,18)=$ $60.9, p<.001]$. This parallels the results of Higashiyama (in press) and Higashiyama and Tashiro (1990).

We also compared the small concentric electrodes with each of the unifocal electrodes. The small concentric electrodes generated a significantly larger threshold than did the $5-\mathrm{mm}[t(9)=$ $4.6, p<.001]$ or the $10-\mathrm{mm}[t(9)=4.6, p<.001]$ unifocal electrode. Thus, a larger threshold is obtained with the small concentric electrodes than with the $7-\mathrm{mm}$ unifocal electrodes.

\section{Experiment B}

In Experiment B, we examined how keratin paste reduces the current threshold for the unifocal electrodes. The cathode $5.0 \mathrm{~mm}$ in diameter was placed on the ventral side of the right forearm and the anode $30.0 \mathrm{~mm}$ in diameter was placed on the instep of the right foot. The forearm and instep of the subject were washed with an alcohol solution, and the anode filled with keratin paste was then placed on the instep. The thresholds for single 2-msec pulses were measured under both cathode conditions, with and without paste. Three of the 6 subjects followed the with- and without-paste order, and the other 3 subjects followed the without- and with-paste order. The mean threshold for the cathode with paste $(M=1.52 \mathrm{~mA}, S D=.31 \mathrm{~mA})$ was significantly smaller than that for the cathode without paste $(M=$ $2.00 \mathrm{~mA}, S D=.63 \mathrm{~mA})[t(5)=2.71, p<.05]$.

\section{Discussion}

In summary, (1) the assignment of polarity for the concentric electrodes did not affect the threshold; (2) under the condition without keratin paste, the small concentric electrodes generated a significantly higher threshold than did the 7-mm unifocal electrodes; and (3) the reduction of the threshold by keratin paste was about $.5 \mathrm{~mA}$.

Since the difference in threshold between the unifocal and the concentric electrodes in Experiments 1 and 2 was much larger than the difference in threshold that would be produced by keratin paste alone, it seems that the concentric electrodes provide a higher threshold than the unifocal electrodes, regardless of whether keratin paste is applied. The distance between electrodes is probably a critical factor influencing the threshold. Higashiyama and Tashiro (1983) showed that the threshold is constant for the electrodes separated by $7 \mathrm{~mm}$ or more, but that the threshold increases rapidly as the separation decreases from $7 \mathrm{~mm}$.

(Manuscript received April 27, 1992; revision accepted for publication December 11, 1992.) 